\title{
Effect of Low-intensity Pulsed Ultrasound (LIPUS) with Different Frequency on Bone Defect Healing
}

\author{
Kazuya Monden ${ }^{1,2)}$, Hodaka Sasaki ${ }^{1,2)}$, Masao Yoshinarii') and Yasutomo Yajima ${ }^{1,2)}$ \\ 1) Department of Oral and Maxillofacial Implantology, Tokyo Dental College, Chiba, Japan \\ 2) Division of Oral Implants Research, Oral Health Science Center, Tokyo Dental College, Chiba, Japan \\ (Accepted for publication, February 23, 2015)
}

\begin{abstract}
Low-intensity pulsed ultrasound (LIPUS) is known to promote bone defect healing. The frequency of the LIPUS is known to influence directivity and depth of penetration, but the differences on bone healing remains unknown. This study was to investigate the effect of LIPUS with different frequencies on bone defect healing. Bone defects of $1.6 \mathrm{~mm}$ in diameter were created in femurs of ten-week-old male Long-Evans rats $(\mathrm{n}=36)$. Experimetal groups were exposed LIPUS (intensity: $30 \mathrm{~mW} / \mathrm{cm} 2$, burst width: $200 \mu \mathrm{s}$, time: $15 \mathrm{~min} /$ day) and divided into a low frequency (LF, 1.5 MHz) group and a high frequency (HF, 3.0 MHz) group. Nonexposed LIPUS group were used as control. After 3, 5, 7, and 10 days, femurs were removed and radiological, histomorphological and molecular biological evaluations were conducted. Micro-CT images showed that the depression in cortical bone was reduced in LF and HF groups. 3D bone morphological analysis at 10 days revealed that LIPUS increased cortical bone volume / tissue volume (BV/TV) and decreased BV/TV in the lower layer of cancellous bone $(\mathrm{P}<0.05)$. Histomorphologically, clot retraction was seen in the both LIPUS groups but not in the control group at 3 days. These effects were observed at a deeper layer in the LF group than in the HF group. No significant difference in osteopontin (OPN) gene expression was observed. However, osteocalcin $(\mathrm{OCN})$ gene expression was significantly elevated in the HF group relative to the control group at 10 days $(\mathrm{P}<0.05)$. Immunohistochemical staining revealed that newly formed bone exhibited a positive reaction to OPN and OCN in both LIPUS groups. Healing of the bone defect area was noted in both LIPUS groups, but there was no clear difference in histomorphology between the LF and HF groups. LIPUS frequencies of 1.5 $\mathrm{MHz}$ and $3.0 \mathrm{MHz}$ promote increased cortical bone mass and remodeling of cancellous bone in rat femurs with bone defects.
\end{abstract}

Key words: Low-intensity pulsed ultrasound, Frequency, Bone healing, Dental implant

\section{Introduction}

In recent years, titanium dental implants that restore occlusal function by achieving osseointegration with the jaw bone have come to be widely used as a treatment for tooth missing ${ }^{1}$. With the dental implant treated commonly, it has also become necessary for patients with implant risk factors such as osteoporosis and diabetes to receive implants. Therefore, a large amount of research has been reported on improving aspects of implants such as surface topography and chemistry in order to securely achieve osseointegration more quickly and increase the success rate of implant in these patients ${ }^{2-4)}$. However, although many studies have been conducted on the effects of improving the implant body as graft side, very few studies have been conducted on methods of improving the jaw bone as host side. One method of improving the jaw bone condition is to inject drugs such as bone

Correspondence to: Dr. Hodaka Sakaki, Department of Oral and Maxillofacial Implantology, Tokyo Dental College, 2-9-18 Misaki-cho, Chiyoda-ku, Tokyo, 101-0061 Japan; Phone: +81-3-6380-9257; E-mail: hosasaki@tdc.ac.jp morphogenetic protein-2 $2^{5)}$, fibroblast growth factor $2^{6)}$ and simvastatin $^{7)}$ into the extraction socket, which has been shown to promote bone healing. However, the effectiveness of these methods are based on the drug chemistry, and these drugs present various issues regarding the risk of side effects, the dose, and the limited areas in which they can be used. Therefore, it is necessary to establish a method of promoting extraction socket healing and improving jaw bone condition that is safe for the body.

In the field of orthopedic surgery, low-intensity pulsed ultrasound (LIPUS), a non-invasive technique that causes no drugrelated side effects and utilizes physical stimulation, is used in clinical settings to promote healing of normal ${ }^{8)}$ and intractable ${ }^{9,10)}$ bone fractures. In vitro studies have shown that LIPUS stimulation increases expression of osteoblast differentiation markers and accelerates calcification $^{11-14)}$. Furthermore, in vivo studies have shown that LIPUS promotes healing and increases bone mineral density in fractured rat femurs ${ }^{15,16}$. LIPUS has also been shown to promote bone fracture healing in rat models of osteoporosis ${ }^{17)}$ 


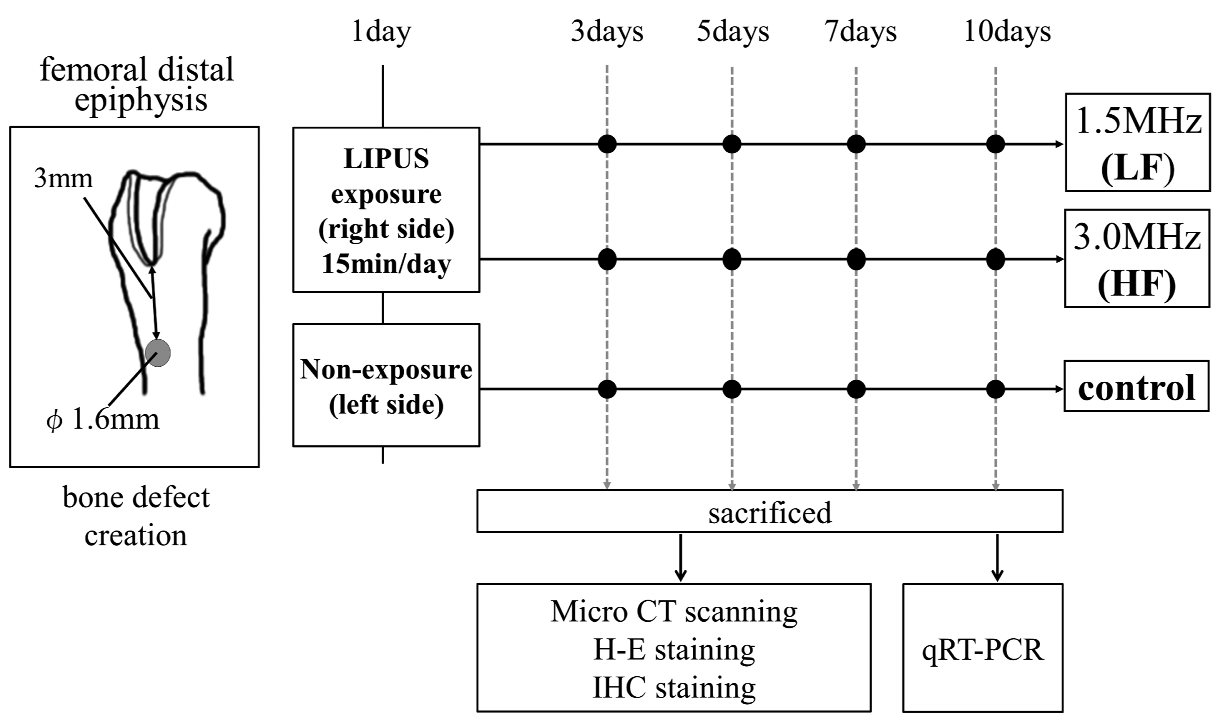

Figure 1. Bone defect model in rat femur and experimental protocol IHC: Immunohistochemical staining, qRT-PCR: quantitative RT-PCR

and diabetes ${ }^{18)}$ known as model of delayed bone healing. In addition, LIPUS is also known to promote bone defect healing in rats of the cranial and tibia bone ${ }^{19,20)}$. Studies in the field of oral implantology have shown that LIPUS exposure improves the contact rate of newly formed bone in implants placed in rabbit femurs $^{21)}$ and promotes the formation of new bone tissue in areas of bone augmentation in the maxillary sinus of rabbits ${ }^{22}$. The findings of these studies indicate that LIPUS is also useful in implant therapy because it promotes achieving osseointegration and extraction socket healing process.

The parameters for LIPUS include intensity, exposure time and frequency. Because LIPUS is a type of ultrasound and its wave is dispersed, scattered, and weakened by tissues ${ }^{23)}$, LIPUS exposure effect is strongly influenced by these parameters. Differences in the intensity of LIPUS, which indicates the strength of the sound waves, are known to contribute to osteocyte differentiation, and optimal parameter is defined ${ }^{11,24)}$. In addition, it has been shown that the healing period can shorten dosedependent LIPUS exposure time ${ }^{25)}$. The frequency of LIPUS is known to contribute to directivity and the depth of penetration. Although the directivity of ultrasonic energy improves at higher frequencies, the depth of penetration decreases. Therefore, in clinical practice, $3 \mathrm{MHz}$ is used for superficial wounds whereas 1 $\mathrm{MHz}$ is used for deep wounds and when there is a large amount of subcutaneous fat ${ }^{23)}$. Thus, if different frequencies of LIPUS could be used in implant therapy to selectively promote healing of cortical and cancellous bone in the jaw bone or to effectively promote bone healing in extraction sockets of different sizes and shapes, it would be a useful method for improving the host side. However, not only have the effects of the frequency of LIPUS on achieving osseointegration and extraction socket healing process, its effects on bone defect healing also remain unstudied.
The purpose of this study is to investigate the effect of low and high frequency LIPUS exposure in the rat femur bone defect healing process by radiological, histomorphological, and molecular biological evaluations.

\section{Materials and Methods}

\section{Surgical bone defect creation and LIPUS stimulation}

Ten-week-old male Long-Evans rats (Sankyo Labo Service Corporation, Inc, Tokyo, Japan; $n=36$ ) were used in this study. After peritoneal injections of pentobarbital sodium (Somnopentyl $^{\circledR}$, Kyoritsu Seiyaku Corporation, Tokyo, Japan; 0.9 $\mu 1 / \mathrm{g}$ ) were administered as general anesthesia. For surgery, the hind legs of the rats were shaved considerably and the outside skin of the distal femur was incised longitudinally, and the femur was exposed by stripping the periosteum. The bone defects were created in both femurs at $3 \mathrm{~mm}$ from the articular surface of the knee joint using motor handpiece (G3, Urawa Corporation, Kuki city, Japan) with a round bur (1.6 mm diameter, Dentsply Maillefer, Ballaigues, Switzerland). The depth of the bone defect was created to reach the opposite side of the cortical bone perforation. After the bone defect was created, the periosteum was replaced and the surgical wound sutured. Starting from one day after bone defect creation, the bone defect area of the right femur was transcutaneously exposed to LIPUS (intensity: $30 \mathrm{~mW} /$ $\mathrm{cm}^{2}$, burst width: $200 \mu \mathrm{s}$, time: $15 \mathrm{~min} /$ day, transducer size: $\mathrm{M}$ [3.2cm diameter], frequency: $1.5 \mathrm{MHz}$ or $3.0 \mathrm{MHz}$ ) with gel as a conductive medium using ST-SONIC (Ito Co, Ltd, Tokyo, Japan). The frequency parameters for LIPUS were low frequency (LF, $1.5 \mathrm{MHz}$ ) and high frequency (HF, 3.0 MHz). The left femurs that composed the non-LIPUS group were used as the control group (Fig. 1). Six samples were taken for radiological and histological evaluation after $3,5,7$, and 10 days $(n=24)$, and three 




Figure 2. Scheme of regions of interest in 3D bone morphological analysis

samples were taken for quantitative RT-PCR after 7 and 10 days $(n=12)$. All experiments were performed according to the Guidelines for the Treatment of Animals at Tokyo Dental College (approval ID: 253002).

\section{X-ray micro-CT}

Rats were sacrificed with pentobarbital sodium after 3, 5, 7 and 10 days, and perfusion fixation was performed with $10 \%$ neutral buffered formalin (Wako Pure Chemical Industries, Osaka, Japan) ( $\mathrm{n}=6$ for each femur). Micro-CT images of bone defect area were taken with the in vivo micro X-ray CT system $\mathrm{R}$ _mCT2 (Rigaku Corporation, Tokyo, Japan). Scanning parameters were as follows: tube voltage, $90 \mathrm{kV}$; tube current, $140 \mu \mathrm{A}$; magnification, $\times 10$; slice width, $20 \mu \mathrm{m}$; and scanning time, $2 \mathrm{~min}$.

\section{Radiological analysis of newly formed bone}

Micro-CT image data at 10 days that had no artifacts were selected (each $n=3$ ) and the 3D structure of newly formed bone was measured using TRI/3D-BON 3D Trabecular bone structure analysis software (Ratoc System Engineering Corporation, Tokyo, Japan). The Region of interest (ROI) was a cylindrical section in the center of the bone defect area that was $1.1 \mathrm{~mm}$ in diameter and contained bone from the top of the cortical bone area of the bone defect to the inner surface of the cortical bone area of the deep part of the bone defect. This ROI was divided into a cortical bone area and a cancellous bone area. The cancellous bone area was further divided into an upper layer and a lower layer (Fig. 2). Bone volume/tissue volume (BV/TV) was used for evaluation and measured 3 times for each samples. The Tukey test was used for statistical processing $(\mathrm{P}<0.05)$.

\section{Histological evaluation}

Samples that had been used for micro-CT image scanning were fixed in $10 \%$ neutral buffered formalin for 7 days and decalcified with $10 \%$ EDTA (pH 7.0-7.5; Wako Pure Chemical Industries,
Osaka, Japan) over 6 days. Paraffin sections of $3 \mu \mathrm{m}$ in thickness were prepared and stained with hematoxylin-eosin (H-E) according to the standard procedure. Histological observations were made using a universal photo microscope (Axiophot2, Carl Zeiss, Oberkochen, Germany).

\section{RNA extraction and quantitative RT-PCR (qRT-PCR)}

The rats were sacrificed with pentobarbital sodium after 7 and 10 days which observed newly bone formation in Histological evaluation, and bone tissue samples for RNA extraction was collected using trephine bar $(2.8 \mathrm{~mm}$ internal diameter, Micro Tech Corp, Tokyo, Japan) from the center of the bone defect area (each $\mathrm{n}=6$ ). Collected bone tissue was kept in RNAlater RNA stabilization reagent (Applied Biosystems, Foster City, CA, USA) and then homogenized (tungsten carbide beads; $5 \mathrm{~mm}$ diameter, $28 \mathrm{~Hz}, 2 \mathrm{~min}$ ) using a Tissue Lyser (QIAGEN, Alameda, CA, USA). Total RNA was extracted from the lysate using an RNeasy ${ }^{\mathbb{R}}$ Mini kit (QIAGEN) according to the manufacturer's protocol and quantified with a NanoDrop ${ }^{\circledR}$ Spectrophotometer ND-1000 (NanoDrop Technologies, Wilmington, DE, USA). The mRNA expression levels of osteopontin (AssayID Rn01449971_g1) and osteocalcin (AssayID Rn00566386_g1) were confirmed by qRTPCR using a TaqMan ${ }^{\circledR}$ MGB probe (Applied Biosystems) and normalized against $\beta$-actin (Applied Biosystems). Total RNA was reverse-transcribed using QuantiTect ${ }^{\circledR}$ Reverse Transcription (QIAGEN), and qRT-PCR was performed with TaqMan ${ }^{\circledR}$ Fast Universal PCR Master Mix (Applied Biosystems) and an ABI 7500 Fast Prism Sequence Detection System (Applied Biosystems) according to the manufacturer's instructions. This quantification for each sample was duplicated, and results were analyzed using the $\Delta \Delta \mathrm{Ct}$ method. Values are expressed as the mean and standard error and were analyzed with the Tukey test.

\section{Immunohistochemical staining}

Paraffin sections of histological evaluation at 10 days were deparaffinized with xylene and rehydrated in ethanol. They were then washed in $10 \mathrm{nmol} / 1$ phosphate-buffered saline (PBS, pH: 7.4 ) and immersed in $0.3 \%$ hydrogen peroxide in ethanol for 30 min to block endogenous peroxidase activity. After the sections were washed in PBS, they were blocked with $3 \%$ normal bovine serum (BSA; Roche Applied Science, Indianapolis, USA). After reacting the sections with the primary antibodies, rabbit antiosteopontin (Millipore Corporation, Billerica, MA, USA; diluted 1:200) and rabbit anti-osteocalcin (BiossInc, MA, USA; diluted $1: 200)$, for 1 hour at room temperature, they were reacted with the secondary antibody, biotinylated anti-rabbit IgG antibody (Histofine MAX-PO [MULTI]; Nichirei, Toyo, Japan), for 30 minutes at room temperature. After washing in PBS, the sections were stained with 3,3'-diaminobenzidine (DAB) (DAB substrate kit, Nichirei, Tokyo, Japan) at room temperature. After 

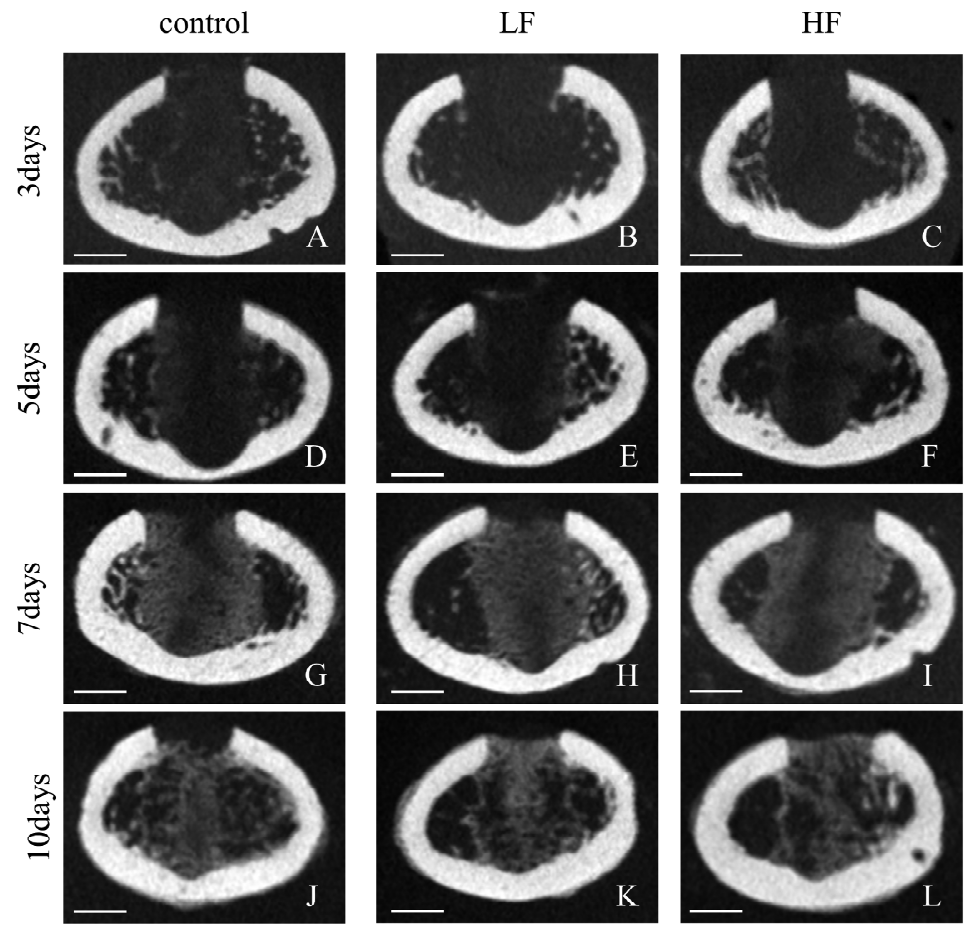

Figure 3. Radiological evaluation of bone defect area by micro-CT scanning Control group: A, D, G, J, Low frequency (LF) group: B, E, H, K, High frequency (HF) group: C, F, I, L, Bar: $1 \mathrm{~mm}$
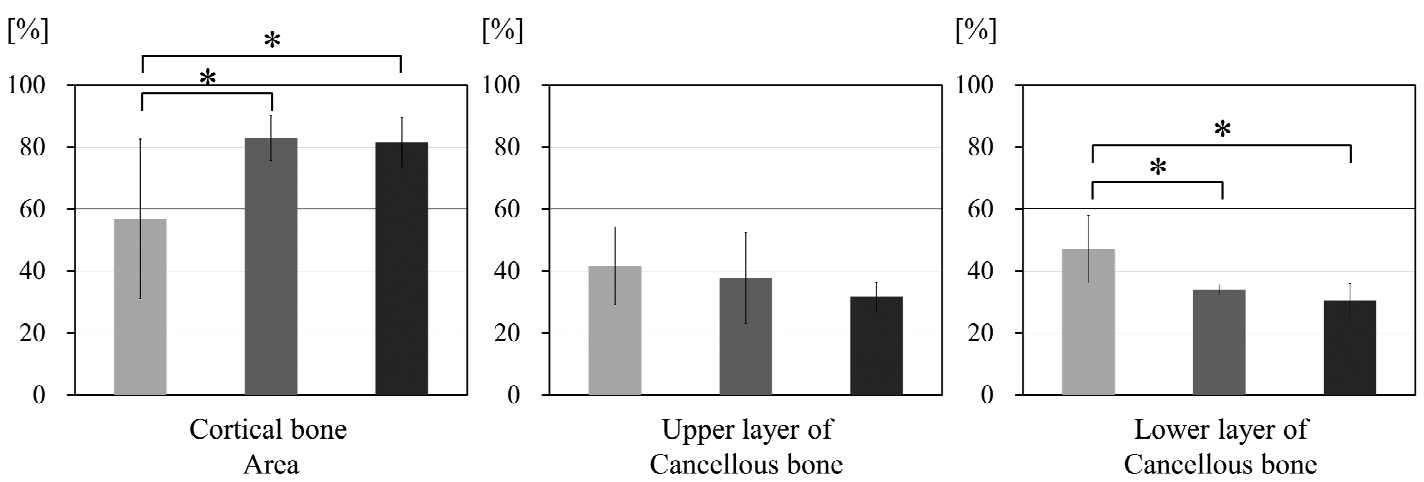

$$
* P<0.05 \square \text { control } \square \mathrm{LF} \quad \square \mathrm{HF}
$$

Figure 4. Measurement of newly formed bone at 10 days by $3 \mathrm{D}$ bone morphological analysis

Mesurement item: Bone Volume / Tissue Volume (BV/TV) In cortical bone, BV/TV was significantly higher in both LIPUS groups (LF and HF) than in the control group $(\mathrm{P}<0.05)$. And at the lower layer of cancellous bone was significantly lower in both LIPUS groups than in the control group $(\mathrm{P}<0.05)$.

counterstaining with a hematoxylin solution, they were dehydrated and enclosed according to the established protocol, and then were observed with a universal photo microscope (Axiophot 2).

\section{Results}

\section{Radiological observation of micro-CT}

Micro-CT images of the bone defect area taken at 3, 5, 7 and 10 days after LIPUS exposure were evaluated (Fig. 3). At 3 days, only radiolucent region that indicated the bone defect were observed (Fig. 3A-C). At 5 days, some radiopaque findings that indicated new bone formation were observed (Fig. 3D-F), but there was no difference among three groups. At 7 days, although growth of newly formed bone and radiopacity were increasing in the bone defect area, there was no difference among all groups (Fig. 3G-I). At 10 days, formation of flat new bone continuous with existing bone was observed in the cortical bone of the LF and HF LIPUS groups, and newly formed bone in cancellous bone defect area was assimilated to existing bone (Fig. 3K, L). In the control group, the cortical bone in the defect area was depressed (Fig. 3J).

\section{$3 D$ bone structural measurement of newly formed bone at 10 days}

$\mathrm{BV} / \mathrm{TV}$ of newly formed bone was calculated (Fig. 4). In cortical bone, BV/TV was significantly higher in both LIPUS groups (LF and HF) than in the control group $(\mathrm{P}<0.05)$. In the upper layer of cancellous bone, there was no significant difference. 
Kazuya Monden et al.: LIPUS with different frequency on Bone Healing
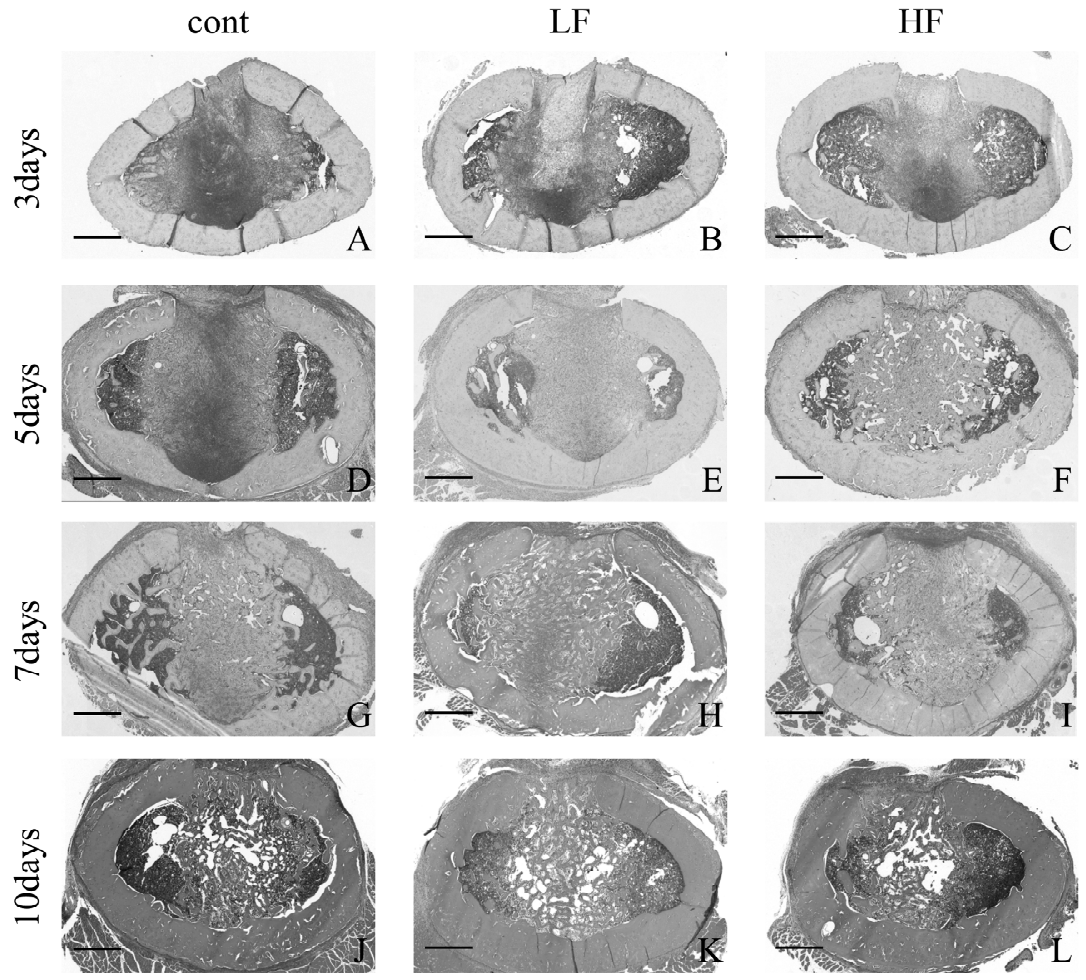

Figure 5. Low maginification of histomorphological evaluation

Low magnification image of $\mathrm{H}-\mathrm{E}$ staining section at $3,5,7$ and 10 days; Control group: A, D, G, J, Low frequency (LF) group: B, E, H, K, High frequency (HF) group: C, F, I, L,

Bar: $1 \mathrm{~mm}$
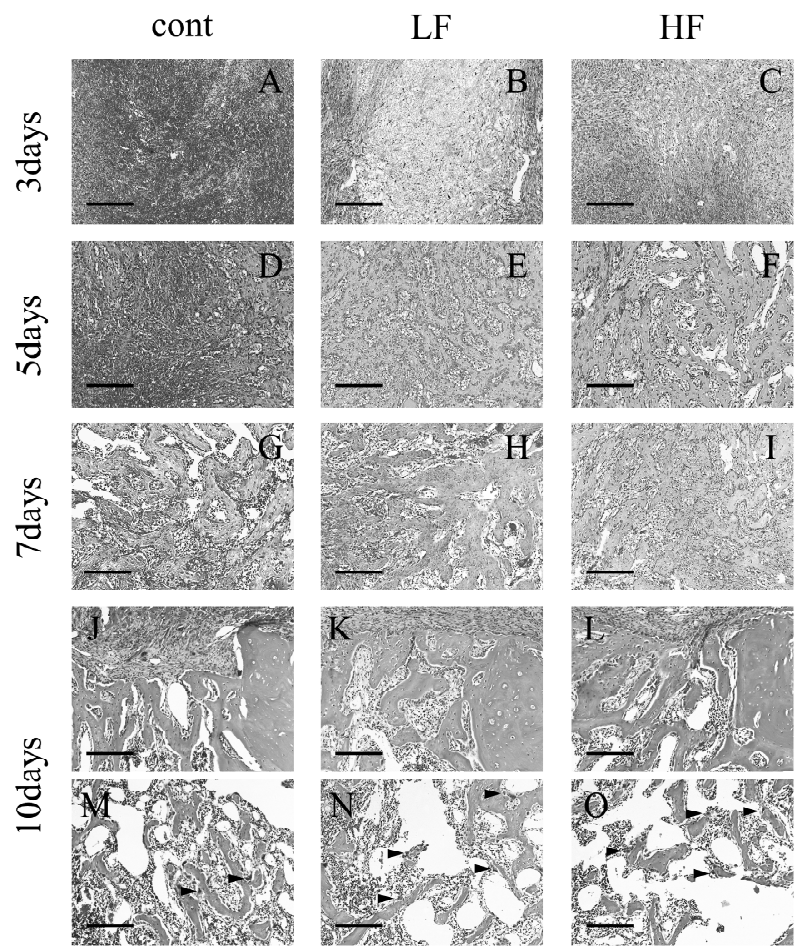

On the other hand, BV/TV at the lower layer of cancellous bone was significantly lower in both LIPUS groups than in the control group $(\mathrm{P}<0.05)$.

\section{Histomorphological evaluation}

Fig. 5 showed low magnification images of H-E staining
Figure 6. High Magnification of histomorphological evaluation High magnication image of $\mathrm{H}-\mathrm{E}$ staining section at 3, 5, 7 and 10 days; central cancellous bone defected area of Control goup: A, D, G, M, Low frequency (LF) group: B, E, H, N, High frequency (HF) group: C, F, I, O, and cortical bone defected area of Control group: J, LF group: K, HF group: L, Bar: 0.2mm, Arrow: osteoclast sections and Figure 6 were high magnification of central cancellous bone defect area (A-I, M-O) and cortical bone defected area (J-L). At 3 days after LIPUS exposure, cortical bone defect and blood clots in bone marrow area were observed in the all groups (Fig. 5A-C). These blood clots were recognized in all layers in the control group (Fig. 6A), whereas blood clot was retracted 

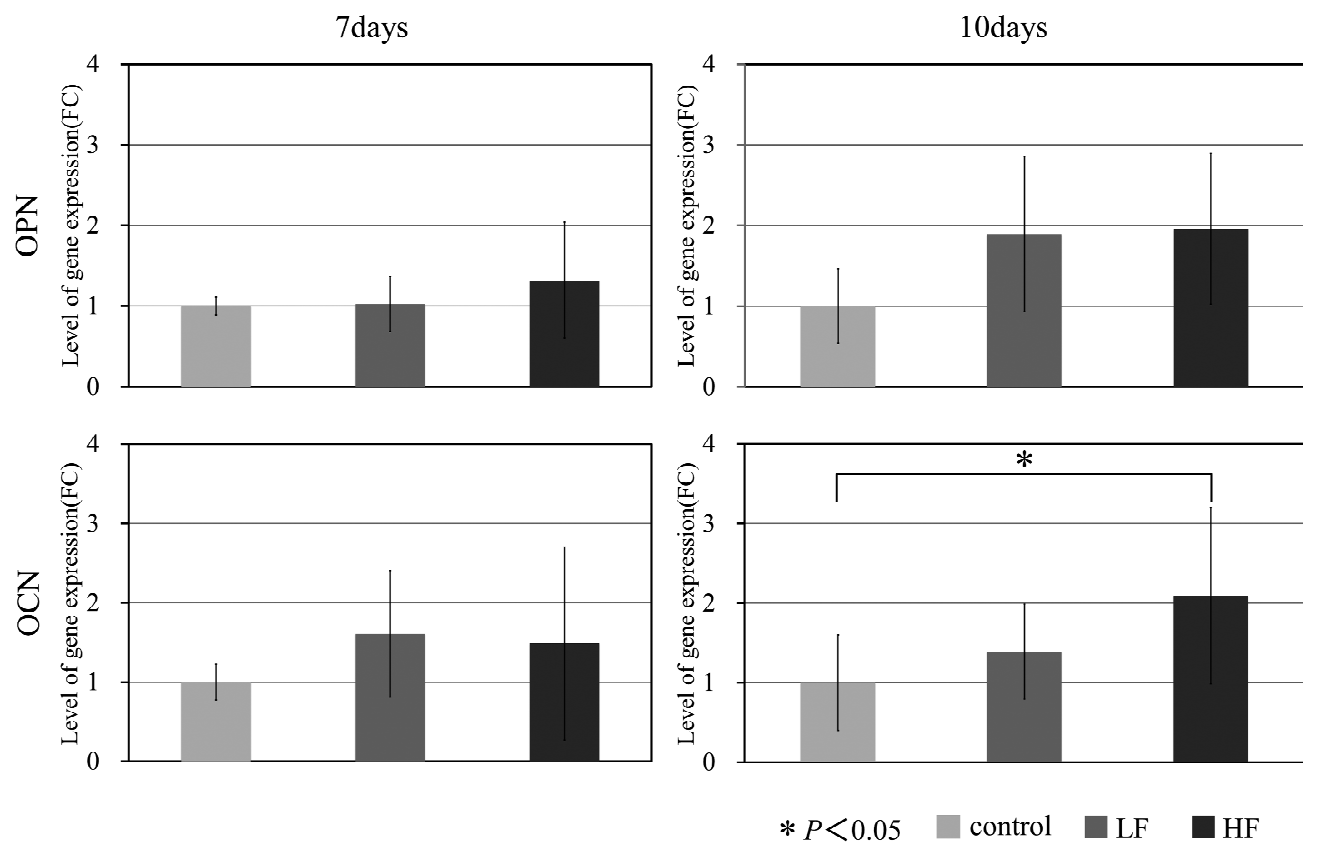

Figure 7. Gene expression quantification for osteopontin (OPN) and osteocalcin (OCN) by quantitative RT-PCR at 7 and 10 days FC: Fold Change
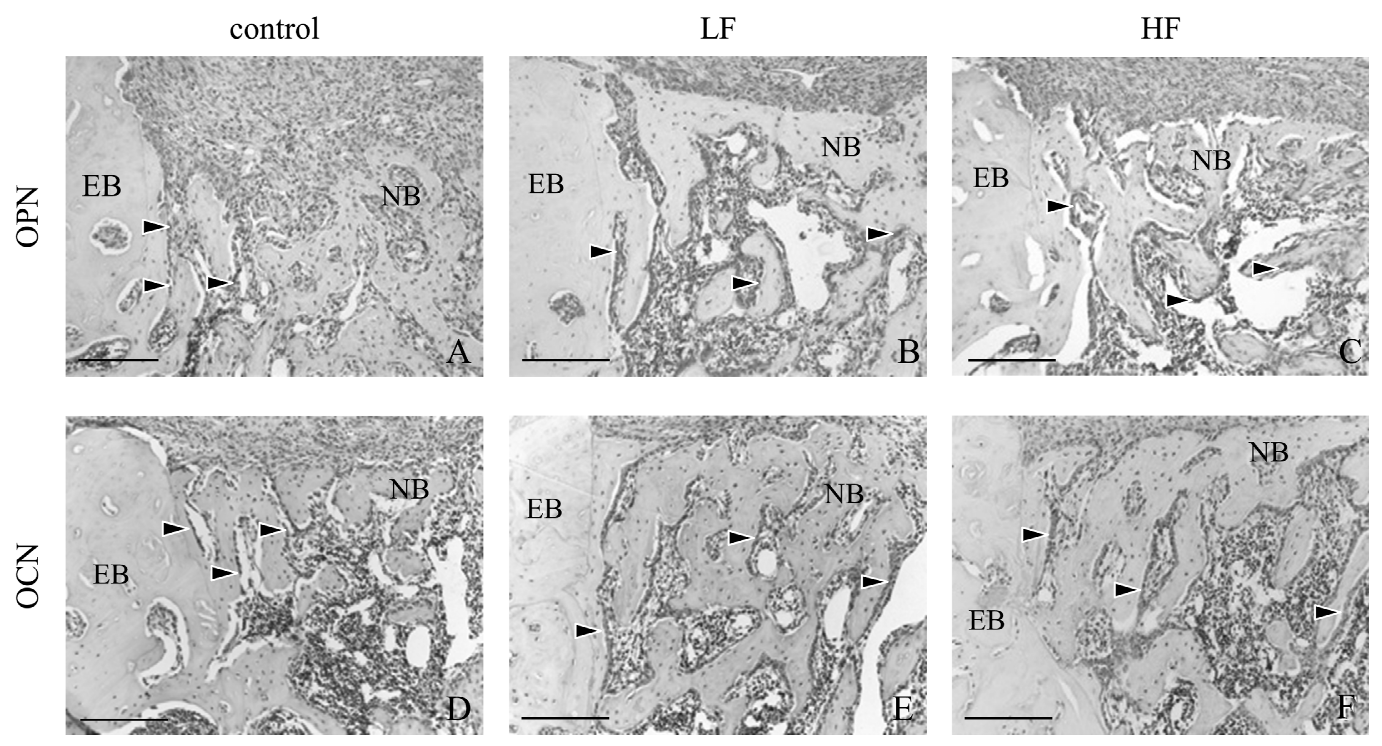

Figure 8. Immunohistochemical staining for osteopontin (OPN) and osteocalcin $(\mathrm{OCN})$ at 10 days

Control group: A, D, Low frequency (LF) group: B, E, High frequency (HF): group: C, F, Bar: $200 \mu \mathrm{m}$, Arrow: positive reaction,

EB: exiting bone, NB: newly formed bone

in both LIPUS groups. In addition, the regression of blood clots in the LF group observed deeper layer area (Fig. 6B) than the HF group (Fig. 6C). At 5 days, there were still blood clots in the control group (Fig. 5D), whereas they were disappeared in both LIPUS groups (Fig. 5E, F). The granulation tissue containing red blood cells and newly bone formation at boundary zone of bone defect area was observed in control groups (Fig. 6D). On the other hands, newly bone formation with osteoblast lining from existing cancellous bone was observed in all layers of the bone defect area in both LIPUS groups (Fig. 6E, F). At 7 days, callus bone was filled in bone defect area in all groups (Fig. 5G-I). The callus bone in central cancellous bone defect area became more mature than at 3 days and capillary vessel formation in connective tissue was observed in all groups (Fig. 6 G-I). At 10 days, densification of newly-formed cortical bone and trabeculation of newly-formed cancellous bone was observed in all groups (Fig. $5 \mathrm{~J}-\mathrm{L}$ ). In cortical bone area, a depression was observed in the control group (Fig. $6 \mathrm{~J}$ ) but not in both LIPUS groups (Fig. 6K, L). In cancellous bone area, capillary formation between trabeculae and bone resorption by osteoclasts (arrowed) were observed in the LIPUS 
Kazuya Monden et al.: LIPUS with different frequency on Bone Healing

groups (Fig. 6N,O) more than control group (Fig. 6M).

\section{QuantitativeRT-PCR (qRT-PCR)}

The gene expression of osteopontin (OPN) and osteocalcin $(\mathrm{OCN})$ was quantified using samples at 7 and 10days (Fig. 7). No significant difference in OPN gene expression was observed in samples taken after 7 or 10 days. OCN gene expression had no significant difference among three groups at 7 days. However, that of HF group significantly increased than control group at 10 days $(\mathrm{P}<0.05)$.

\section{Immunohistochemical staining}

Immunolocalization of OPN (Fig. 8A-C) and OCN (Fig. 8DF) in newly-formed cortical bone at 10 days after LIPUS exposure was investigated by immunohistochemical staining. Immunoreaction of OPN in the control group was recognized newly formed bone adjacent to stump of existed bone (Fig. 8A). However, positive reaction of OPN in osteoblasts around the newly formed bone was seen not only stump of existed bone side, but also center side of bone defect area in both LPUS groups (Fig. $8 \mathrm{~B}, \mathrm{C}$ ). A positive immunoreaction to $\mathrm{OCN}$ was observed in newly formed bone at the boundary with existing bone in the control group (Fig. 7D). In both LIPUS groups (Fig. 7E, F), a positive reaction to $\mathrm{OCN}$ was observed in osteoblasts around newly formed bone from the stump of existing bone to the middle of the bone defect area.

\section{Discussion}

In this study, the effects of different frequencies of LIPUS exposure on bone defect healing process were compared in rat femur bone defect models by radiological, histomorphological, and molecular biological evaluations. LIPUS is a type of ultrasound energy that passes through living tissues ${ }^{23}$ and is known to promote healing of fractures and bone defects ${ }^{8,15,19)}$. The parameters for LIPUS include intensity, exposure time and frequency. Some previous studies have examined the effects of differences in LIPUS intensity on bone tissue ${ }^{11,26)}$ and reported an intensity of $30 \mathrm{~mW} / \mathrm{cm}^{2}$ LIPUS promotes osteoblast differentiation in vitro ${ }^{14)}$ and promotes fracture healing in a rat femur model in vivo ${ }^{15)}$. It was also revealed LIPUS exposure at $30 \mathrm{~mW} / \mathrm{cm}^{2}$ promoted fracture healing and was safety in the clinical study ${ }^{8,27,28)}$. From these results, the United States Food and Drug Administration currently recommends that an intensity of $30 \mathrm{~mW} /$ $\mathrm{cm}^{2}$ be used when using LIPUS for human bone fracture. An intensity of $30 \mathrm{~mW} / \mathrm{cm}^{2}$ was used in this study as well.

At 10 days LIPUS exposure, the reduction of depression, increase of $\mathrm{BV} / \mathrm{TV}$ and immunoreactions for OPN and OCN in newly formed cortical bone were observed in newly formed bone in both LIPUS groups (LF and HF), however not in the control group. LIPUS is known to promote cell proliferation and increased bone differentiation marker expression in cultured rat bone marrow derived stromal cells ${ }^{12)}$ and human periosteal cells ${ }^{29)}$, and to accelerate healing process of bone fracture and bone defect. Moreover, Yoshida A et al. ${ }^{30}$ ) found that LIPUS reduced the depression and accelerated densification of cortical bone in bone defect areas of mouse femurs by promoteing periosteal cell differentiation. Naruse K et al. ${ }^{31)}$ also reported that LIPUS increased osteocalcin expression in periosteal cells and promotes periosteal cell-derived stem cells differentiation into osteoblasts in organ culture of rat femurs. These results suggested that LIPUS exposure matured newly formed bone in cortical bone area by increasing bone differentiation marker; OCN and OPN and reduced the depression by promoting periosteal cell differentiation.

On the other hands, BV/TV were decreased in the lower layer of cancellous bone in both LIPUS groups compared to control group. The process of bone healing divided into 4 stages; hematoma formation stage, fibrocartilaginous callus formation stage, bony callus formation stage, and bone remodeling stage. The samples taken at 10 days after bone defect creation in this study showed matured callus bone and partial bone resorption by osteoclast in both LIPUS group and it was indicated that the latter part of the bone healing process, which is transition between the bony callus formation stage and bone remodeling stage. In the some studies of LIPUS exposure with distraction osteogenesis has been shown to promote bone remodeling by callus maturation and bone resorption ${ }^{32,33)}$. Therefore, it was suggested, LIPUS exposure brought about callus resorption in lower layer of cancellous bone by accelerating the transition into the bone remodeling stage in this study.

The frequency range for LIPUS is considered to be $0.75-3$ $\mathrm{MHz}^{23)}$. In this study, the frequencies were set to $1.5 \mathrm{MHz}$ for the LF group and 3.0 MHz for the HF group. In the histomorphological evaluation at 3 days after LIPUS exposure, blood clot retraction and accompanying granulation tissue was seen in both LIPUS groups but not in the control group. Blood clot retraction tended to occur at a deeper level in the LF group than in the HF group. LIPUS has been reported to activate macrophages ${ }^{15)}$ and increase the expression of platelet-derived growth factor ${ }^{34)}$, fibroblast growth factor ${ }^{35)}$, and vascular endothelial growth factor ${ }^{36)}$ which were known to involved in the transition between hematoma formation stage and fibrocartilaginous callus formation stage. It is also known that lower frequencies of LIPUS have a greater depth of penetration ${ }^{23}$. Based on these result, it is suggested the early stages of bone healing was faster in the LF group because the effects of LIPUS penetrated more deeply than in the HF group.

In samples taken after 10 days, gene expression of OCN was significantly higher in the HF group than in the control group. It is known that directivity improves as the frequency of LIPUS increases. In this study, gel was applied to the skin around rat femurs as a conductive medium, and the femurs were exposed to 
LIPUS for $15 \mathrm{~min} /$ day using a transducer with a diameter of 3.2 $\mathrm{mm}$. Therefore, the direction of propagation was not always constant, and it is suggested that differences in the directivity of LIPUS could have influenced the effects observed in samples taken after 10 days. However, there is not recognized histomorphological differences in bone healing were observed between the LF and HF groups at 10 days in this study. The depth of penetration of LIPUS is known as $1-2 \mathrm{~cm}$ for the $3 \mathrm{MHz}$ frequency and 3-5 cm for the $1 \mathrm{MHz}$ frequency ${ }^{23)}$. Thus, differences caused by the depth of penetration would not appeared in this study model using rat femurs even if width of soft tissue includes skin and muscle (approximately $2 \mathrm{~cm}$ ), and the diameter of the femur (approximately $3 \mathrm{~cm}$ ) were combined because the total depth is still less than $5 \mathrm{~cm}$.

In this study, 1.5 MHz (LF) and 3.0 MHz (HF) frequencies of LIPUS both promoted healing in the bone defect area. This indicates that LIPUS could be useful in implant therapy to promote healing of the extraction socket before implant placement and achieving implant neck region of osseointegration. Additionally, LIPUS exposure in oral cavity is difficult to maintain a standard direction of propagation, it is better to use a high frequency to improve directivity in areas where the depth of penetration is 5 $\mathrm{cm}$ or less, including the cortical bone of the alveolar crest and bone surrounding the implant neck region. However, the effect of the frequency of LIPUS on the cancellous area of the human jaw bone, where the depth of penetration is greater than $5 \mathrm{~cm}$, will need to be examined in future studies.

In conclusion, bone defects in rat femurs, LIPUS frequencies of $1.5 \mathrm{MHz}$ and $3.0 \mathrm{MHz}$ promote increased cortical bone mass and remodeling of cancellous bone.

\section{References}

1. Dierens M, Vandeweghe S, Kisch J, Nilner K and De Bruyn H. Long-term follow-up of turned single implants placed in periodontally healthy patients after 16-22 years radiographic and peri-implant outcome. Clin Oral Implants Res 23: 197-204, 2012

2. Fischer K and Stenberg T. Prospective 10-year cohort study based on a randomized controlled trial (RCT) on implantsupported full-arch maxillary prostheses. Part 1: sandblasted and acid-etched implants and mucosal tissue. Clin Implant Dent Relat Res 14: 808-815, 2012

3. Buser DA, Schroeder A, Sutter F and Lang NP, The new concept of ITI hollow- cylinder and hollow-screw implants: Part 2. Clinical aspects, indications, and early clinical results. Int J Oral Maxillofac Implants 3: 173-181, 1988

4. Omar OM, Lennerås ME, Suska F, Emanuelsson L, Hall JM, Palmquist A and Thomsen P. The correlation between gene expression of proinflammatory markers and bone formation during osseointegration with titanium implants. Biomaterials
32: 374-386, 2011

5. Fiorellini JP, Howell TH, Cochran D, Malmquist J, Lilly LC, Spagnoli D, Toljanic J, Jones A and Nevins M. Randomized study evaluating recombinant human bone morphogenetic protein-2 for extraction socket augmentation. J Periodontol 76: 605-613, 2005

6. Lalani Z, Wong M, Brey EM, Mikos AG, Duke PJ, Miller MJ, Johnston C and Montufar-Solis D. Spatial and temporal localization of FGF-2 and VEGF in healing tooth extraction sockets in a rabbit model. J Oral Maxillofac Surg 63: 15001508,2005

7. Wu Z, Liu C, Zang G, and Sun $\mathrm{H}$. The effect of simvastatin on remodelling of the alveolar bone following tooth extraction. Int J Oral Maxillofac Surg 37: 170-176, 2008

8. Heckman JD, Ryaby JP, McCabe J, Frey JJ and Kilcoyne RF. Acceleration of tibialfracture-healing by non-invasive, lowintensity pulsed ultrasound. J Bone Joint Surg Am 76: 2634, 1994

9. Lerner A, Stein H, and Soudry M. Compound high-energy limb fractures with delayed union: our experience with adjuvant ultrasound stimulation (exogen). Ultrasonics 42: 915-917, 2004

10. Rutten S, Nolte PA, Korstjens CM, van Duin MA, and KleinNulend J. Low-intensity pulsed ultrasound increases bone volume, osteoid thickness and mineral apposition rate in the area of fracture healing in patients with a delayed union of the osteotomized fibula. Bone 43: 348-354, 2008

11. Gleizal A, Li S, Pialat JB and Beziat JL. Transcriptional expression of calvarial bone after treatment with lowintensity ultrasound: an in vitro study. Ultrasound Med Biol 32: 1569-1574, 2006

12. Sena K, Leven RM, Mazhar K, Sumner DR and Virdi AS. Early gene response to low-intensity pulsed ultrasound in rat osteoblastic cells. Ultrasound Med Biol 31: 703-708, 2005

13. Jiang $\mathrm{T}, \mathrm{Xu} \mathrm{T}, \mathrm{Gu} \mathrm{F}, \mathrm{Chen} \mathrm{A}, \mathrm{Xiao} \mathrm{Z}$ and Zhang D. Osteogenic effect of low intensity pulsed ultrasound on rat adiposederived stem cells in vitro. J Huazhong Univ Sci Technol Med Sci 32: 75-81, 2012

14. Suzuki A, Takayama T, Suzuki N, Sato M, Fukuda T and Ito K. Daily low-intensity pulsed ultrasound-mediated osteogenic differentiation in rat osteoblasts. ActaBiochimBiophys Sin (Shanghai) 41: 108-115, 2009

15. Wang SJ, Lewallen DG, Bolander ME, Chao EY, Ilstrup DM and Greenleaf JF. Low intensity ultrasound treatment increases strength in a rat femoral fracture model. J Orthop Res 12: 40-47, 1994

16. Warden SJ, Fuchs RK, Kessler CK, Avin KG, Cardinal RE and Stewart RL. Ultrasound produced by a conventional therapeutic ultrasound unit accelerates fracture repair. Phys 
Ther 86: 1118-1127, 2006

17. Cheung WH, Chin WC, Qin L and Leung KS. Low intensity pulsed ultrasound enhances fracture healing in both ovariectomy-induced osteoporotic and agematched normal bones. J Orthop Res 30: 129-136, 2012

18. Gebauer GP, Lin SS, Beam HA, Vieira P and Parsons JR. Low-intensity pulsed ultrasound increases the fracture callus strength in diabetic BB Wistar rats but does not affect cellular proliferation. J Orthop Res 20: 587-592, 2002

19. Hasuike A, Sato S, Udagawa A, Ando K, Arai Y and Ito K. In vivo bone regenerative effect of low-intensity pulsed ultrasound in rat calvarial defects. Oral Surg Oral Med Oral Pathol Oral Radiol Endod 111: 12-20, 2011

20. Renno AC, Fávaro-Pípi E, Fernandes K, Tim C and Ribeiro DA. Ultrasound therapy modulates osteocalcin expression during bone repair in rats. Ultrasonics 52: 111-116, 2012

21. Liu Q, Liu X, Liu B, Hu K, Zhou X and Ding Y. The effect of low-intensity pulsed ultrasound on the osseointegration of titanium dental implants. Br J Oral Maxillofac Surg 50: 244-250, 2012

22. Takebe H, Nakanishi Y, Hirose Y and Ochi M. Effect of low intensity pulsed ultrasound stimulation on sinus augmentation in rabbits. Clin Oral Implants Res 25: 735 741,2014

23. Speed CA. Therapeutic ultrasound in soft tissue lesions. Rheumatology (Oxford) 40: 1331-1336, 2001

24. Angle SR, Sena K, Sumner DR andVirdi AS. Osteogenic differentiation of rat bone marrow stromal cells by various intensities of low-intensity pulsed ultrasound. Ultrasonics 51: 281-288, 2011

25. Chan CW, Qin L, Lee KM, Cheung WH, Cheng JC and Leung KS. Dose-dependent effect of low-intensity pulsed ultrasound on callus formation during rapid distraction osteogenesis. J Orthop Res 24: 2072-2079, 2006

26. Lavandier B, Gleizal A and Béra JC. Experimental assessment of calvarial bone defect re-ossification stimulation using low-intensity pulsed ultrasound. Ultrasound Med Biol 35: 585-594, 2009

27. Kristiansen TK, Ryaby JP, McCabe J, Frey JJ and Roe LR. Accelerated healing of distal radial fractures with the use of specific, low-intensity ultrasound. A multicenter, prospective, randomized, double-blind, placebo-controlled study. J Bone Joint Surg Am79: 961-973, 1997

28. Cook SD, Ryaby JP, McCabe J, Frey JJ, Heckman JD and Kristiansen TK. Acceleration of tibia and distal radius fracture healing in patients who smoke. Clin Orthop Relat Res1: 198-207, 1997

29. Leung KS, Cheung WH, Zhang C, Lee KM and Lo HK. Low intensity pulsed ultrasound stimulates osteogenic activity of human periosteal cells. Clin Orthop Relat Res 418: 253-259, 2004

30. Yoshida A, Sasaki H, Furuya Y, Yoshinari M and Yajima S. Effect of low-intensity pulsed ultrasound on bone-healing process in murine low-turnover osteoporosis model. J Hard Tissue Biology 22: 301-310, 2013

31. Naruse K, Mikuni-Takagaki Y, Urabe K, Uchida K and Itoman M. Therapeutic ultrasound induces periosteal ossification without apparent changes in cartilage, Connect Tissue Res 50: 55-63, 2009

32. El-Mowafi $\mathrm{H}$ and Mohsen M. The effect of low-intensity pulsed ultrasound on callus maturation in tibial distraction osteogenesis. Int Orthop 29: 121-124, 2005

33. Shimazaki A, Inui K, Azuma Y, Nishimura N and Yamano Y. Low-intensity pulsed ultrasound accelerates bone maturation in distraction osteogenesis in rabbits. J Bone Joint Surg Br 82: 1077-1082, 2000

34. Ito M, Azuma Y, Ohta T and Komoriya K. Effects of ultrasound and 1,25-dihydroxyvitamin D3 on growth factor secretion in co-cultures of osteoblasts and endothelial cells. Ultrasound Med Biol 26: 161-166, 2000

35. Reher P, Doan N, Bradnock B, Meghji S and Harris M. Effect of ultrasound on the production of IL-8, basic FGF and VEGF. Cytokine 11: 416-423, 1999

36. Katano M, Naruse K, Uchida K, Mikuni-Takagaki Y, Takaso $\mathrm{M}$, Itoman $\mathrm{M}$ and Urabe K. Low intensity pulsed ultrasound accelerates delayed healing process by reducing the time required for the completion of endochondral ossification in the aged mouse femur fracture model. Exp Anim 60: 385395,2011 
J.Hard Tissue Biology Vol. 24(2):189 - 198, 2015 